BNL-75067-2005-CP

\title{
Remarks on the Maximum Entropy Method Applied to Finite Temperature Lattice $Q C D$
}

\author{
Takashi Umeda \\ Brookhaven National Laboratory, Upton, New York, 11973, USA \\ Hideo Matsufuru \\ High Energy Accelerator Research Organization (KEK), Tsukuba 305-0801, Japan \\ Presented at the $23^{\text {rd }}$ International Symposium on Lattice Field Theory \\ Dublin, Ireland \\ July 25-30, 2005
}

October 2005

XXX Physics Department XXX
Brookhaven National Laboratory
P.O. Box 5000
Upton, NY 11973-5000
www.bnl.gov

Notice: This manuscript has been authored by employees of Brookhaven Science Associates, LLC under Contract No. DE-AC02$98 \mathrm{CH} 10886$ with the U.S. Department of Energy. The publisher by accepting the manuscript for publication acknowledges that the United States Government retains a non-exclusive, paid-up, irrevocable, world-wide license to publish or reproduce the published form of this manuscript, or allow others to do so, for United States Government purposes. 


\section{DISCLAIMER}

This report was prepared as an account of work sponsored by an agency of the United States Government. Neither the United States Government nor any agency thereof, nor any of their employees, nor any of their contractors, subcontractors, or their employees, makes any warranty, express or implied, or assumes any legal liability or responsibility for the accuracy, completeness, or any third party's use or the results of such use of any information, apparatus, product, or process disclosed, or represents that its use would not infringe privately owned rights. Reference herein to any specific commercial product, process, or service by trade name, trademark, manufacturer, or otherwise, does not necessarily constitute or imply its endorsement, recommendation, or favoring by the United States Government or any agency thereof or its contractors or subcontractors. The views and opinions of authors expressed herein do not necessarily state or reflect those of the United States Government or any agency thereof. 


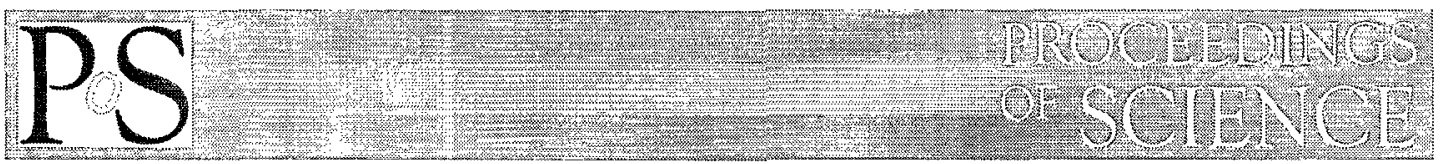

\title{
Remarks on the Maximum Entropy Method applied to finite temperature lattice QCD
}

\author{
Takashi Umeda*i \\ Brookhaven National Laboratory, Upton, New York, 11973, USA \\ E-mail: tumeda@quark.phy.bnl.gov

\section{Hideo Matsufuru} \\ High Energy Accelerator Research Organization (KEK), Tsukuba 305-0801, Japan \\ E-mail: hideo.matsufuru@kek.jp
}

\begin{abstract}
We make remarks on the Maximum Entropy Method (MEM) for studies of the spectral function of hadronic correlators in finite temperature lattice QCD. We discuss the virtues and subtlety of MEM in the cases that one does not have enough number of data points such as at finite temperature. Taking these points into account, we suggest several tests which one should examine to keep the reliability for the results, and also apply them using mock and lattice QCD data.
\end{abstract}

XXIIIrd International Symposium on Lattice Field Theory

25-30 July 2005

Trinity College, Dublin, Ireland

${ }^{*}$ Speaker.

${ }^{\dagger}$ A footnote may follow. 


\section{Introduction}

In the lattice QCD, most properties of hadrons are extracted from the hadronic correlation functions. The spectral function (SPF) has particular importance, since it may contain information beyond the stable ground state and a few excited states which can be extracted by standard fitting techniques. Recent development of analysis techniques such as the maximum entropy method (MEM) [1] have enabled direct extraction of SPFs from numerical data of lattice QCD simulation. At zero temperature, MEM has been successfully reproduced correct features of the SPFs $[1,2]$.

At finite temperature, we can calculate the SPF from the thermal green functions in principle using the same procedure as at zero temperature [3,4]. In particular, charmonium states have drawn much attention, since they probe the QCD plasma state through the changes of their properties $[5,6]$, and hence are potential signal of the formation of quark gluon plasma in the heavy ion collision experiments [7]. Several groups have studied the SPF of charmonium in finite temperature lattice QCD using MEM $[8,9,10]$ and their results indicate persistent $J / \psi$ state even above $T_{c}$. MEM has also been extensively applied to various areas of lattice field theories [11].

While MEM is a powerful tool to extract SPF, it has intrinsic subtlety when applied to lattice QCD data of correlators. In this paper, we point out how each ingredient of MEM analysis causes such subtlety, focusing on an application to the correlators at finite temperature. In the next section, we consider general problems of MEM, and then in Sect. 3 describe particular problem at finite temperature caused by short extent in the temporal direction. Details of these analysis were presented in Ref. [8].

\section{Maximum entropy method}

\subsection{Outline of MEM}

First we briefly summarize the outline of MEM basically following Ref. [1], which reviews in detail MEM applied to data of lattice QCD simulation. We obtain the SPF, $A(\omega)$, from the given lattice result for the correlator, $C(t)$, by solving the inverse problem,

$$
C(t)=\int_{0}^{\infty} d \omega K(t, \omega) A(\omega)
$$

where the (continuum type) kernel $K(t, \omega)$ is given by

$$
K(t, \omega)=\frac{e^{-\omega t}+e^{-\omega\left(N_{t}-t\right)}}{1-e^{-N_{t} \omega}}
$$

To extract the SPF $A(\omega)$, MEM maximizes a functional $Q(A ; \alpha)=\alpha S[A]-L[A] . L[A]$ is the usual likelihood function, and minimized in the standard $\chi^{2}$ fit. The Shannon-Jaynes entropy $S[A]$ is defined as

$$
S[A]=\int_{0}^{\infty} d \omega\left[A(\omega)-m(\omega)-A(\omega) \log \left(\frac{A(\omega)}{m(\omega)}\right)\right] .
$$

The function $m(\omega)$ is called the default model function, and should be given as a plausible form of $A(\omega)$. At the last stage of calculation the parameter $\alpha$ can be integrated out by a weighted average of prior probability for $\alpha$. 

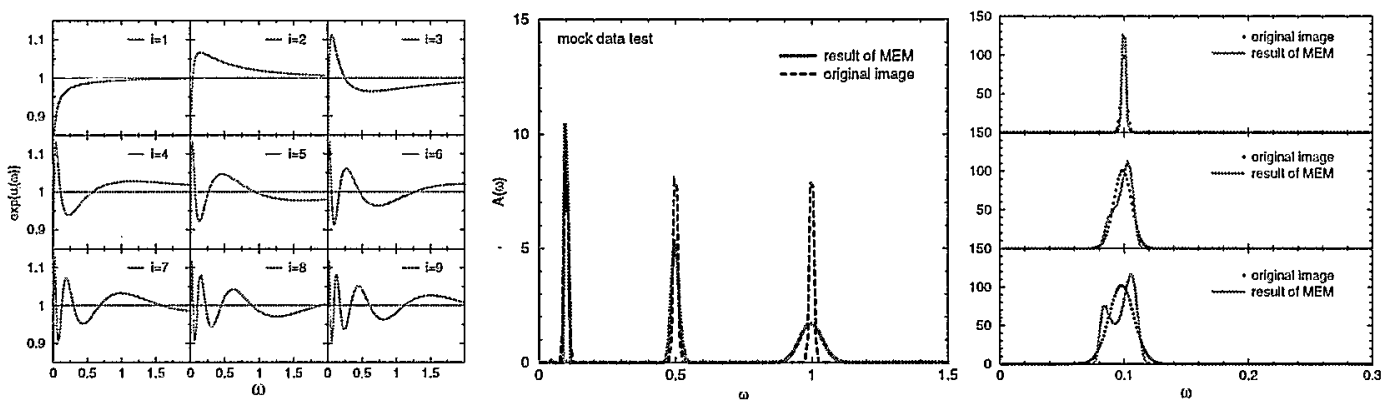

Figure 1: Left panel: Samples of eigenfunction for the kernel, Eq. (2.2). The eigenfunction $\exp \left(u_{i}\right)$ corresponds to a part of Eq. (2.5). Center and right panels: The results of mock data analysis. The dotted line is an original SPF and the solid line is reconstructed result by MEM.

\subsection{Singular Value Decomposition}

In the maximization step of $Q(A ; \alpha)$ the singular value decomposition of the kernel $K(t, \omega)$ is usually used [1]. ${ }^{1}$ Then the SPF is represented as a linear combination of the eigenfunctions of $K(t, \omega)$ :

$$
A(\omega)=m(\omega) \exp \left\{\sum_{i=1}^{N_{s}} b_{i} u_{i}(\omega)\right\}
$$

where $N_{s}$ is the number of eigenfunctions, $b_{i}$ are parameters, and $u_{i}(\omega)$ the eigenfunction of the kernel $K(t, \omega)$. The number of degrees of freedom of $A(\omega)$ is accordingly reduced to the number of data points of the correlator. Although $b_{i}$ can in principle be determined uniquely from the data without introducing an entropy term, the small eigenvalues of $K(t, \omega)$ lead to a singular behavior of the SPF; hence truncation of the terms is practically required, i.e. $N_{s}$ may be less than the number of data points [13]. In MEM, the entropy term stabilizes the problem and guarantees an unique solution for the coefficients of the eigenfunctions [1].

An outstanding feature of Eq.(2.4) is that it can be fitted to generic shape without restriction to specific forms such as a sum of poles. However, the resolution of course depends on the number of degree of freedom in Eq. (2.4), and also on $\omega$. An example of eigenfunctions is displayed in the left panel of Fig. 1. This figure indicates that the resolution of the function becomes worse in large $\omega$ region, because the superposed functions do not have enough variation.

This feature of the eigenfunction is also shown in the mock data analysis. Center panel of Figure 1 shows the original (input) SPF and the reconstructed SPF from the correlator which is constructed by the original SPF with a random Gaussian noise. The original SPF has three peaks of the same width and hight at each $\omega$. When the noise of correlator is not so small, the reconstructed SPF does not agree with the original one; there is a tendency that the peak becomes broader than the original one at high energy region. The peak positions are correctly reproduced in this case.

The right panel of Figure 1 shows more interesting example. When the width of a peak is narrow, MEM reproduces the shape rather well. However, for a case of large width, MEM fails to reproduce the shape of the original peak.

\footnotetext{
${ }^{1}$ Analysis of MEM without singular value decomposition was examined in Ref. [12].
} 


\subsection{Default model function}

As mentioned in section 2.1, MEM needs a default model function to define the entropy term $Q$. Since small difference between a trial SPF and default model function makes the entropy term large, the default model function strongly affects the result of MEM when the quality of data is not sufficient. Therefore the default model function should include only reliable information we know beforehand. If not so, there is a risk the result might be controlled by hand.

In the case of QCD, prior knowledge for SPFs is not so many, e.g. positivity and perturbative behavior at high energy region. In the case of point correlators, a natural choice of the default model function is the asymptotic behavior of the meson correlators at large $\omega$ in perturbation theory. We should remember, however, that such an asymptotic behavior is not observed in practical simulation because of the finite lattice cutoff.

The risk caused by lack of reliable default model function is reduced by the quality of data. In fact, this perturbative form has been successfully applied to problems at zero temperature $[1,2]$. When we do not have good quality of data and reliable default model function, we have to check, at least, a default model function dependence to estimate an systematic uncertainty for the results.

\section{Application to finite temperature lattice QCD}

Since a temporal lattice extent, $N_{t}$, is restricted to $1 / T a_{t}$ on finite temperature lattices, it is usually difficult to keep good quality of data as compared with zero temperature. Therefore we have to check the reliability of the results.

One of good checks is to apply the method to the zero temperature data in the same condition as at finite temperature. To extract the SPF at finite temperature, at least, we should successfully reproduce the zero temperature SPF from the zero temperature correlator but the number of data points restricted to $1 / T a_{t}$. We show these checks with our lattice data, which was obtained on an anisotropic lattice with $\beta=6.10$ and the renormalized anisotropy $\xi=4$ having the spatial cutoff $a_{\sigma}^{-1}=2.030(13) \mathrm{GeV}[8]$.

We apply MEM to the correlator with restricted numbers of degree of freedom. The results with two types of such restrictions are displayed in Fig. 2. The left panel shows the dependence of the result on $t_{\max }$, the maximum $t$ of the correlator used in the analysis. This case corresponds to the situation at $T>0$. MEM fails to reproduce even the lowest peak for $t_{\max } \leq 16$. The center panel shows the results when one alternatively skips several time slices in the analysis. This case corresponds to the coarsening of the temporal lattice spacing. Even for $t_{s e p}=8$ for which the number of data point is 6, MEM at least reproduces the correct lowest peak position while the resolution is not enough. These result indicate that the physical region of the correlator as well as the number of the degrees of freedom is important for MEM to work correctly. The required region of $C(t)$ in the above analysis is $t_{\max }>O(0.5 \mathrm{fm})$, which is not fulfilled around $T \sim T_{c}$. This situation may be improved by smeared operators. The left panel shows the results of MEM for the correlator with smeared operator. It is stable under the above two kinds of restriction for $t_{\max }$ of interest; at least the lowest peak position is correctly reproduced.

Next we show the default model function dependence for a correlator with the smeared operator at finite temperature in Fig. 3. In this analysis we adopt the default model function of 

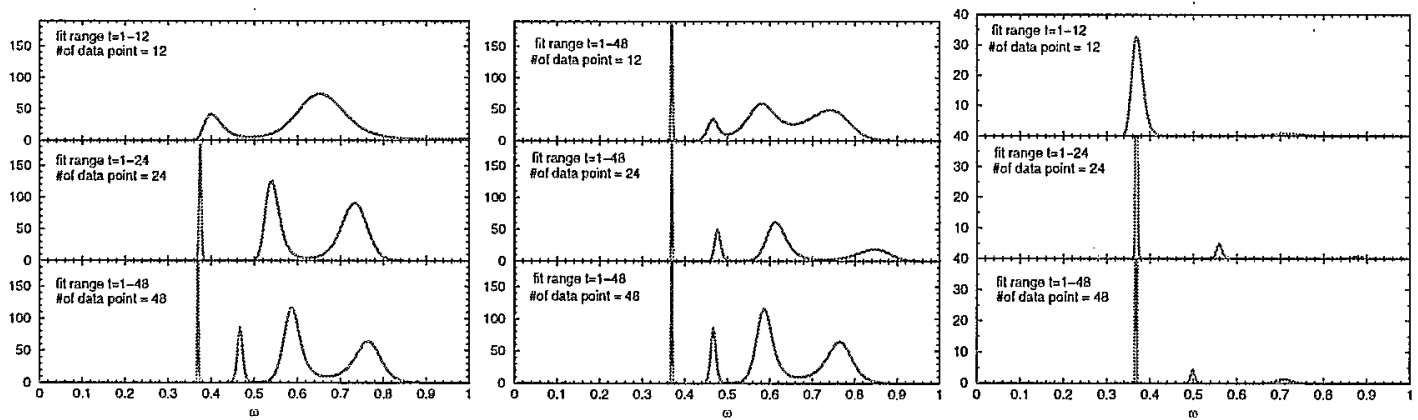

Figure 2: Tests of MEM for restricting number of degree of freedom in the zero temperature correlator. The first two panels show the results with a point operator, and the last is with a smeared operator.

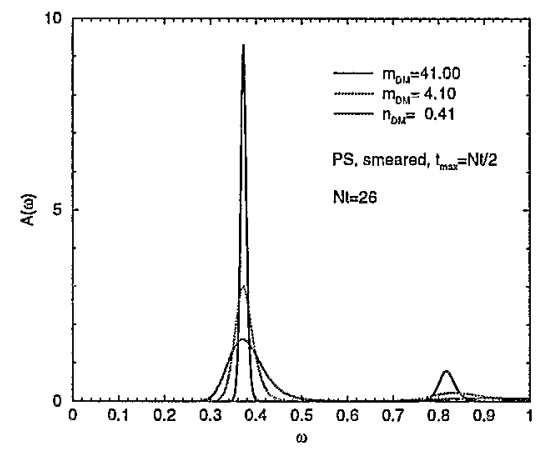

Figure 3: The default model function dependence of SPF for a correlator with the smeared operator at finite temperature.

$m(\omega)=m_{D M} \omega^{2}$, where $m_{D M}$ is determined by the perturbative asymptotic behavior of large $\omega$ region. As mentioned in Sect.2.3, since the default model function is not justified for lattice QCD simulation, we observe the default model function dependence in order to estimate a systematic uncertainty of the MEM results. In Figure 3 we change the $m_{D M}$ by factor 10, 1 and 0.1 respectively. The peak position is stable while the width is rather sensitive to such change of the parameter $m_{D M}$. These results indicate that it is difficult to discuss width of SPFs quantitatively based on MEM analysis.

\section{Conclusion}

In our previous paper [8] we have concluded the MEM is not sufficient for quantitative study of the SPF from our lattice data even if some smeared operators are adopted. If we roughly know the shape of SPFs, standard $\chi^{2}$ fit (or constrained curve fitting [14]) is rather appropriate for quantitative studies. Therefore we used MEM to find a rough image (fit-form) of SPFs and performed $\chi^{2}$ fit (or constrained curve fitting) with this functional form, such as multi Breit-Wignar type function, for more quantitative estimate of the width of the peak. In conclusion, although MEM is powerful tool to extract the SPFs from correlators, we have to use it carefully taking its subtlety into account. 
The simulation has been done on NEC SX-5 at Research Center for Nuclear Physics, Osaka University and Hitachi SR8000 at KEK (High Energy Accelerator Research Organization). T.U has been supported by the JSPS Research Fellowships for Young Scientists.

\section{References}

[1] Y. Nakahara, M. Asakawa and T. Hatsuda, Phys. Rev. D 60, 091503 (1999) [arXiv:hep-lat/9905034]. M. Asakawa, T. Hatsuda and Y. Nakahara, Prog. Part. Nucl. Phys. 46, 459 (2001) [arXiv:hep-lat/0011040].

[2] T. Yamazaki et al. [CP-PACS Collaboration], Phys. Rev. D 65, 014501 (2002) [arXiv:hep-lat/0105030].

[3] A.A. Abrikosov, L.P.Gor'kov, and Dzyaloshinskii, Sov. Phys. JETP 36(9), 636 (1959); E.S. Fradkin, Sov. Phys. JETP 36(9), 912 (1959).

[4] P. de Forcrand et al. [QCD-TARO Collaboration], Phys. Rev. D 63, 054501 (2001) [arXiv:hep-lat/0008005]. T. Umeda, R. Katayama, O. Miyamura and H. Matsufuru, Int. J. Mod. Phys. A 16, 2215 (2001) [arXiv:hep-lat/0011085].

[5] T. Hashimoto, K. Hirose, T. Kanki and O. Miyamura, Phys. Rev. Lett. 57, 2123 (1986).

[6] T. Matsui and H. Satz, Phys. Lett. B 178, 416 (1986).

[7] M. C. Abreu et al. [NA50 Collaboration], Phys. Lett. B 477, 28 (2000).

[8] T. Umeda, K. Nomura and H. Matsufuru, Eur. Phys. J. C37S1 (2004) 9. [arXiv:hep-lat/0211003].

[9] M. Asakawa and T. Hatsuda, Phys. Rev. Lett. 92, 012001 (2004) [arXiv:hep-lat/0308034].

[10] S. Datta, F. Karsch, P. Petreczky and I. Wetzorke, J. Phys. G 30, S1347 (2004) [arXiv:hep-lat/0403017].

[11] K. Sasaki, S. Sasaki and T. Hatsuda, Phys. Lett. B 623, 208 (2005) [arXiv:hep-lat/0504020]. M. Imachi, Y. Shinno and H. Yoneyama, Prog. Theor. Phys. 111, 387 (2004) [arXiv:hep-lat/0309156]. N. Ishii and H. Suganuma, Nucl. Phys. Proc. Suppl. 129, 581 (2004) [arXiv:hep-lat/0309102]. T. Yamazaki and N. Ishizuka, Phys. Rev. D 67, 077503 (2003) [arXiv:hep-lat/0210022]. I. Wetzorke and F. Karsch, arXiv:hep-lat/0008008.

[12] H. R. Fiebig, Phys. Rev. D 65 (2002) 094512 [arXiv:hep-lat/0204004].

[13] For pioneering works, QCD-TARO Collaboration, Ph. de Forcrand et al., Nucl. Phys. B (Proc. Supp1.) 63, 460 (1998); E. G. Klepfish, C. E. Creffield, E. R. Pike, ibid., 655 (1998).

[14] G. P. Lepage et al., Nucl. Phys. B (Proc.Suppl.) 106 12(2002). 